\title{
Modeling of Short Channel MOSFET Devices and Analysis of Design Aspects for Power Optimisation
}

\author{
Kiran Agarwal Gupta, Dinesh K. Anvekar, and Venkateswarlu V.
}

\begin{abstract}
The advancement in silicon material processes technology in the manufacture of 45nm MOSFET has extended Moore's Law for some more years. Low power CMOS circuit design has become a challenge due to variations in design parameters caused as a result of short channel effects at deep submicron levels for technology nodes below $1 \mu \mathrm{m}$. In this paper, we have analyzed the design aspects for short channel devices by method of transistor modeling and further simulations have been carried out using Virtuoso cadence Simulator. The technology nodes considered here are $180 \mathrm{~nm}$ and $45 \mathrm{~nm}$ technology since fabrication of $180 \mathrm{~nm}$ uses conventional process technology and $45 \mathrm{~nm}$ uses new innovations in process technology. The aim of this paper is to bring out parameter variability issues related to different process technologies and find solutions for power optimization at design level for CMOS circuits.
\end{abstract}

Index Terms - Short channel effects, CMOS, technology node; Threshold variation, low power, scaling.

\section{INTRODUCTION}

The never ending demand of miniaturization and low power has led to a new dimension in Integrated circuit EraDeep Submicron technology and Nano Technology. But continuous shrinking in channel length has posed new challenges such as short channel effects, high leakage current and static power dissipation. As the transistor scaling continued, fundamental limits of sizing of gate insulator reached and transistor could not be scaled any further. Intel's $45 \mathrm{~nm}$ CMOS technology is the first in the world to replace polysilicon to new high-k dielectric material. Gordon More has called "the biggest change in transistor technology since 1960s" [1]. The insulating material silicon oxide between the transistor's gate and the channel was replaced by hafnium oxide which reduced the leakage current between gate and the channel. But other leakage currents still dominate the chips at smaller nodes giving rise to a need of fundamental change of transistor structure. The other two ways to control the leakage of short channel transistors are ultrathin body silicon-on-insulator or UTB SOI and FinFET or Tri-Gate

Manuscript received March 5, 2013; revise May 8, 2013. This work was supported in part by the United Technologies limited and Honeywell Technology Solution Labs..

Kiran Agarwal Gupta is with Dayananda Sagar College of Engineering in the dept. of E\&C as Associate Professor.Bangalore-560078, India.(e-mail:jpkiran9@gmail.com,g.kiran@ieee.org).

Dinesh Anvekar is with Honeywell Technology Solution Labs as Six Sigma Specialist for Research and Innovations Bangalore-560078, India (e-mail: Dinesh.Anvekar@honeywell.com).

V. Venkateswarlu is with United Technologies Limited working as professor and Principal of VTU Extension Centre.Bangalore-560022, India (e-mail:vwarlu@utltraining.com) devices [2]. Though designers are working on $30 \mathrm{~nm}$ and 20 $\mathrm{nm}$ nodes, it will require new fabrication techniques and has its own limitations and challenges to face ahead. Thus the low power circuit design for high performance can be still explored for existing foundries of $180 \mathrm{~nm}-45 \mathrm{~nm}$ technology nodes.

In this paper we have presented simulation results of 180 $\mathrm{nm}$ and $45 \mathrm{~nm}$ nMOS transistor. Here we have studied and compared short channel Effects (SCE) and other design problems like leakage currents for both the technology nodes. We have also explored the limitations and proposed the possibilities of using these SCE for design of low power CMOS circuits.

This paper is divided into four sections. Section I gives the introduction to the latest scaling trends and the advancement in process technology to overcome SCE. Section II gives a brief overview of the power dissipation concerns for short channel devices. In section III we have presented the simulation results of $180 \mathrm{~nm}$ and $45 \mathrm{~nm}$ devices for study of transistor behavior with respect to sub threshold currents and OFF state leakage currents. Section IV presents the threshold voltage model for short channel devices and experimental results for comparison of both the technologies for SCE and its impact on $\mathrm{V}_{\mathrm{th}}$. Last section is the conclusion of the work.

\section{Static Power Dissipation Due to Scaling OF DEVICES}

Designing of a CMOS circuit above deep submicron levels involved a trade-off between chip area, performance and dynamic power dissipation. Now, deep submicron level circuit designs also have to deal with new design issues called as short channel effects (SCE) and this has led to innovation of new design techniques both at all levels of abstraction from floor planning to device fabrication.

\section{A. Power Dissipation in Short Channel Devices}

As seen from (1) and (2), the performance and operating speed of analog or digital circuits is directly dependent on the supply voltage [3].

$$
P_{d y n}=\alpha C_{L} V_{D D}^{2} f o
$$

where,

$\alpha=$ Switching activity of transistors

$C_{L} \quad=$ Load capacitance

$V_{D D} \quad=$ Supply voltage

$F \quad=$ Operating frequency

$P_{d y n}$ is the power dissipation when the circuit is working or 
in active mode and $P_{\text {static }}$ is the power dissipation when the circuit is not in active mode. $P_{d y n}$ results from charging and discharging of the capacitors due to switching activity of the transistor. But continuous scaling of Metal Oxide Semiconductor field effect transistors (MOSFETS) to deep submicron levels has delivered lesser area and low capacitance effects leading to negligible dynamic power dissipation. Also miniaturization has led to the performance gain, cost reduction, and portability for semiconductor chip applications.

\section{B. Static Power Dissipation in Short Channel Devices}

Static power dissipation is an important factor to be considered for deep submicron chips since it dominates deep submicron devices and increases with shrinking technology node. Equation (2) represents static power dissipation.

$$
P_{\text {static }}=I_{\text {Leakage }} V_{D D}
$$

where $I_{\text {leakage }}$ is the leakage current flowing through different nodes of the circuit in inactive state. The main cause of leakage currents for short channel devices is the non-uniform doping under the gate region and thinner oxide layer which cause more leakages such as subthreshold current leakage, tunneling gate leakage current and OFF state leakage current. Since portable devices and hand-held devices remain in standby mode for longer duration, the stand-by leakage current has become the major design issue. Also, practically it has been observed that decreasing supply voltages for short channel devices has not helped in overall power consumption in circuits. This is due to the fact that at shorter channels dynamic power becomes negligible, but due to rise in static leakage currents, static power dissipation raises making power dissipation almost equal for the CMOS circuits both in $\mathrm{ON}$ and OFF states of the device.

\section{Regions of Operation in MOSFET}

The general behavior of a short channel MOSFET can be explained with Fig.1. The overall operation of a MOSFET has been classified into four regions based on variation of $\mathrm{ON}$ resistance $R_{\text {out }}$ as $V_{D S}$ is increased. The first region of operation is triode or linear region. This region represents channel current when $V_{D S}$ is less than $V_{g s}$ and the output resistance $R_{\text {out }}$ is low.

- Drain characteristics

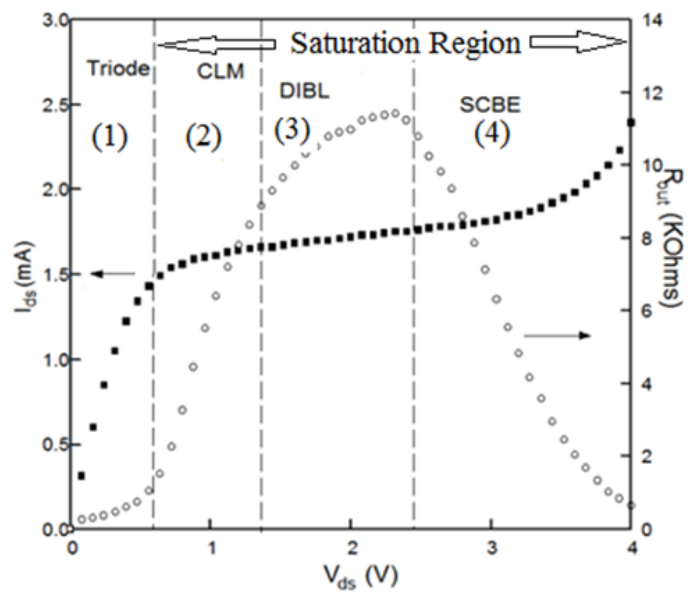

Fig. 1. MOSFET behavior showing $R_{\text {out }}$ and regions of operation [4]
The region of operation above threshold voltage, where channel current becomes constant is called as saturation region which can be further divided in three parts- region 2 , region 3 and region 4 . In the second region channel current has weaker dependence on drain voltage and is dominated by channel length modulation. Region 3 is dominated by DIBL effect wherein threshold voltage has a strong dependence on drain voltage leading to unpredicted variations in circuit performance and has maximum $R_{\text {out }}$. The fourth region is called as Substrate current induced body effect (SCBE) which reduces $R_{\text {out }}$ as shown in figure. When drain voltage is very large (Electric field > $0.1 \mathrm{mV} / \mathrm{cm}$ ), some electrons coming from source will be energetic enough to cause impact ionization increasing substrate current. Thus, $I_{s u b}$ will increase exponentially with drain voltage causing an increase in the drain current and this is also called as impact ionizations and this state of electrons is termed as "HOT" electrons.

\section{OPERATION OF MOSFETS IN SUBTHRESHOLD REGION}

The region of operation below threshold voltage is called as subthreshold region of operation. This region forms very important part of this study as most of the leakage currents flow in this region causing static power dissipation in short channel devices. Equation (3) gives the subthreshold current for short channel devices [4].

$$
I_{S u b}=I_{0}\left[1-\exp \left(-\frac{V_{d s}}{V_{t}}\right)\right] \cdot \exp \left(\frac{V_{g s}-V_{t h}-V_{o f f}{ }^{\prime}}{n V_{T}}\right)
$$

where,

$$
\begin{gathered}
I_{0}=\mu \frac{W}{L} \sqrt{\frac{q \xi_{i} N_{D}}{2 \emptyset_{S}}} \cdot V_{T}^{2} \\
\mathrm{~V}_{\text {off }}{ }^{\prime}=\mathrm{V}_{\mathrm{OFF}}+\mathrm{V}_{\mathrm{OFFL}} / \mathrm{L}_{\mathrm{eff}}
\end{gathered}
$$

where,

$n \quad=$ Sub threshold slope factor $(1<\mathrm{n}<3)$

$I_{O} \quad=$ Process dependent parameter and also depends on device geometry

$W / L=$ is the width to length ratio of MOS device

$V_{g s} \quad=$ Gate to Source voltage

$V_{T}=$ Thermal Voltage, equal to $\mathrm{k}_{\mathrm{B}} \mathrm{T} / \mathrm{q}$,

Short channel effect is the variation of threshold voltage with respect to the channel length. The impact of SCE can be summarized as Sub threshold current, Drain Induced Barrier Lowering (DIBL), Gate Induced Drain lowering (GIDL), Channel length modulation (CLM) and velocity saturation. . In a MOSFET device channel current is dependent on the gate voltage and the drain voltage. Thus the transistor modeling and the behavior can be well understood by I-V characterisayion.

\section{A. Sub-Threshold Leakage Current}

Sub-threshold current, $I_{\text {sub }}$ is the channel current which occurs when $V_{g s}<V_{t h}$ i.e. for gate voltage lesser than threshold voltage of a transistor. This is the main cause for leaky transistors and static power dissipation in short channel devices. It has been investigated that for $180 \mathrm{~nm}$ N-channel 
device, drain current exists for even $V_{g s}$ below 0 Volts as shown in Fig. 2. Though threshold voltage of transistor is $V_{t h}=530 \mathrm{mV}$, "OFF" state leakage current of $20 \mathrm{pA}$ exist even for gate voltage of 0 volts as sown in table I. The range of threshold current is found to be $20 \mathrm{pA}$ to $16 \mu \mathrm{A}$. This accounts for high leakage current in the device and leads to major static power consumption. This current can add up in complex CMOS circuits posing a design challenge for low power circuit design.

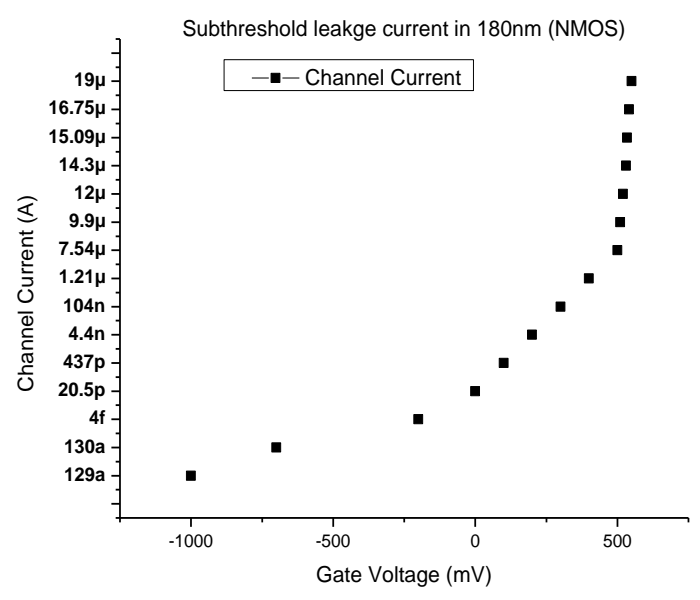

Fig. 2. Threshold voltage curve for 180nm nMOS Device

In case of $180 \mathrm{~nm}$ transistor, $I_{d s}$ increases exponentially and the curve resembles any other silicon transistor as shown in Fig. 2. The comparison between sub threshold current for $180 \mathrm{~nm}$ transistor and $45 \mathrm{~nm}$ transistor has been done in Fig. 3. It can be observed that leakage and "OFF" state current is more for $45 \mathrm{~nm}$ transistor compared to $180 \mathrm{~nm}$. These figures show that sub threshold voltage curve is more linear in case of $180 \mathrm{~nm}$ compared to $45 \mathrm{~nm}$ device. It has been investigated that that $I_{\text {sub }}$ increases linearly for $180 \mathrm{~nm}$ device, but follows a "U" curve for $45 \mathrm{~nm}$ device as shown below.

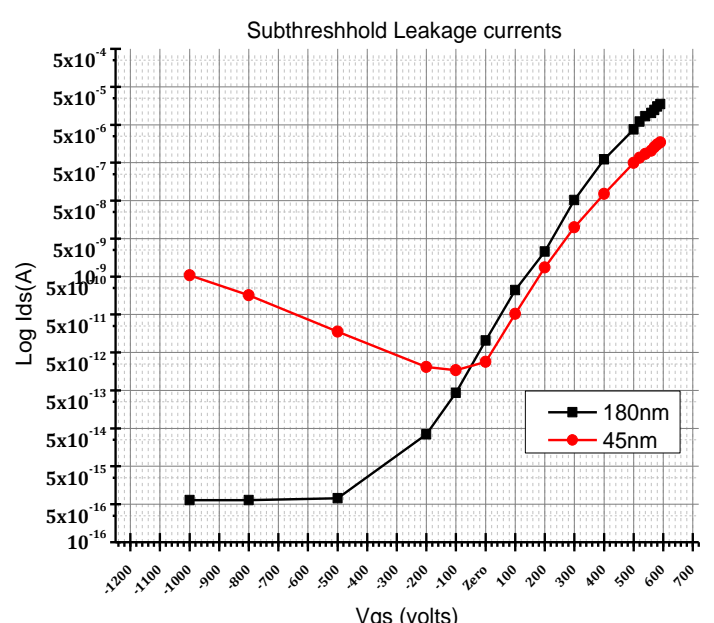

Fig. 3. Sub-Threshold current for $180 \mathrm{~nm}$ and $45 \mathrm{~nm}$ nMOS device

\section{B. “OFF” State Leakage Current}

Table I gives the readings taken from simulation carried out for both devices. The readings have been taken both for $180 \mathrm{~nm}$ and $45 \mathrm{~nm}$ technology nodes for $V_{g s}$ ranging from negative $1000 \mathrm{mV}$ to $+590 \mathrm{mV}$ at $\mathrm{V}_{\mathrm{DS}}=2 \mathrm{~V}$. Fig.4 shows the plot of simulation result of $45 \mathrm{~nm}$ device for $V_{g s}$ Vs. $I_{d s}$, below threshold region. The points M0, M, M2 and M3 give the trace of various points on the plot. Point M1 is measured at. $=-1.96$ with drain current as high as $1.61 \mu \mathrm{A}$. This indicates presence of large "OFF" state leakage current in case of $45 \mathrm{~nm}$ device compared to $180 \mathrm{~nm}$. This current is almost equal to drain current when the device is in saturation region. As $45 \mathrm{~nm}$ process technology is the most advanced technology node today, with best available techniques applied at process level, it is required to mitigate these leakage currents at circuit level. This has been achieved by circuit designers at circuit level by keeping device cut-off from the ground by inserting switches, power-gating techniques and sleep transistor between circuit and power/ground rails.

TABLE I: SUBTHRESHOLD REGION CURRENT IN 180NM AND 45 NM NMOS

\begin{tabular}{lll|l|}
\hline \multicolumn{3}{c}{ DEVICE } \\
\hline \hline $\boldsymbol{V}_{g s}(\boldsymbol{m} \boldsymbol{V})$ & $\boldsymbol{I}_{\boldsymbol{d}}(\boldsymbol{A})$ for 180nm & $\boldsymbol{I}_{\boldsymbol{d}}(\boldsymbol{A})$ for $\mathbf{4 5} \mathbf{n m}$ \\
\hline-1000 & $129 \mathrm{E}-17$ & $1.082 \mathrm{E}-9$ \\
-800 & $129 \mathrm{e}-17$ & $324 \mathrm{E}-12$ \\
-500 & $143 \mathrm{E}-17$ & $35.2 \mathrm{E}-12$ \\
-200 & $70 \mathrm{E}-15$ & $4.13 \mathrm{E}-12$ \\
-100 & $86 \mathrm{E}-15$ & $3.42 \mathrm{E}-12$ \\
0 & $20.5 \mathrm{E}-12$ & $5.66 \mathrm{E}-12$ \\
100 & $437 \mathrm{E}-12$ & $104 \mathrm{E}-12$ \\
200 & $4.55 \mathrm{E}-9$ & $1.74 \mathrm{E}-9$ \\
300 & $1.04 \mathrm{E}-9$ & $19.74 \mathrm{E}-9$ \\
400 & $1.218 \mathrm{E}-6$ & $150 \mathrm{E}-9$ \\
500 & $7.54 \mathrm{E}-6$ & $985 \mathrm{E}-9$ \\
520 & $12 \mathrm{E}-6$ & $1.35 \mathrm{E}-6$ \\
540 & $16.7 \mathrm{E}-6$ & $1.7 \mathrm{E}-6$ \\
560 & $20.5 \mathrm{E}-6$ & $2.07 \mathrm{E}-6$ \\
570 & $24.6 \mathrm{E}-6$ & $2.55 \mathrm{E}-6$ \\
580 & $30.1 \mathrm{E}-6$ & $3 \mathrm{E}-6$ \\
590 & $35 \mathrm{E}-6$ & $3.4 \mathrm{E}-6$ \\
\hline \hline
\end{tabular}

Though leakage current is more for $45 \mathrm{~nm}$ device in below zero volts, there exists more leakage current for $180 \mathrm{~nm}$ transistor in the sub-threshold region. This improvement in $45 \mathrm{~nm}$ device has been achieved by change in process material for fabrication of gate which reduces gate leakage current at high $V_{D S}$ by reducing depletion width under drain.

An interesting behavior is observed in case of $45 \mathrm{~nm}$ $\mathrm{N}$-channel MOSFET in Fig. 4. The characteristic curve with $V_{g s}$ Vs. $I_{d s}$ shows the plot of behavior of $45 \mathrm{~nm}$ device below threshold voltage that is $-2 \mathrm{~V}$ to $560 \mathrm{mV}$. The graph show a $\mathrm{U}$ curve showing more leakage at negative and zero voltage, then increases slowly and turns around $240 \mathrm{mV}$. It is proposed that, this high leakage region can be considered for ultra low power circuit designing with low input signals. But to avoid leakages threshold voltage has been increased to about $570 \mathrm{mV}$ by using different gate process technology. This limits ultra low power applications as transistor works in saturation region only above $600 \mathrm{mV}$. Thus, this leakage current of $45 \mathrm{~nm}$ device still remains a great challenge for circuit designers for low power applications. 


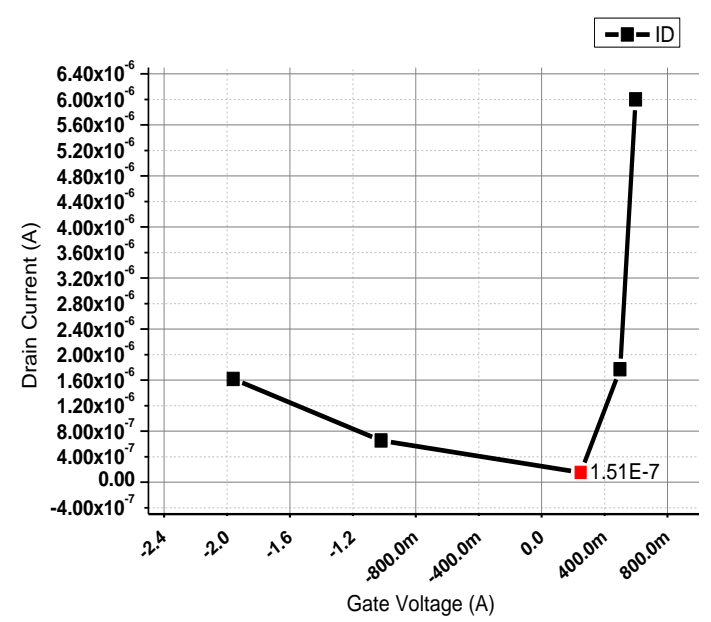

Fig. 4. U curve for $45 \mathrm{~nm}$ transistor in subthreshold region.

As seen from above figure, there exist higher leakage current below zero volts than between $0-\mathrm{V}_{\text {th }}$ volts (about $580 \mathrm{mV}$ )

\section{Threshold Voltage Modeling}

MOSFETs have both process dependent and design dependent parameters. Threshold Variation is the process dependent parameter and plays an important role in CMOS circuit design. Threshold voltage is a process dependent parameter and remains constant for long channel devices i.e. for $\mathrm{L} \geq 1 \mu \mathrm{m}$. But as discussed above, short channel devices show $V_{t h}$ variation due to short channel effects. In short channel devices, $V_{t h}$ also depend on the device parameters like channel length, channel width and drain voltage.

\section{A. Threshold Voltage Equation for Long Channel Devices}

The Threshold Voltage, $V_{T O}$, is defined as the gate potential $V_{G}$ at which the surface potential $\Phi_{\mathrm{s}}$ changes by $2 \Phi_{\mathrm{F}}$, i.e. the surface becomes strongly inverted [5].

$$
V_{T O}=\left[\emptyset_{G C}-\frac{q N_{O X}}{C_{O X}}\right]+\left[-2 \emptyset_{F}-\frac{Q_{B O}}{C_{O X}}\right]+\frac{q N_{1}}{C_{O X}}
$$

where,

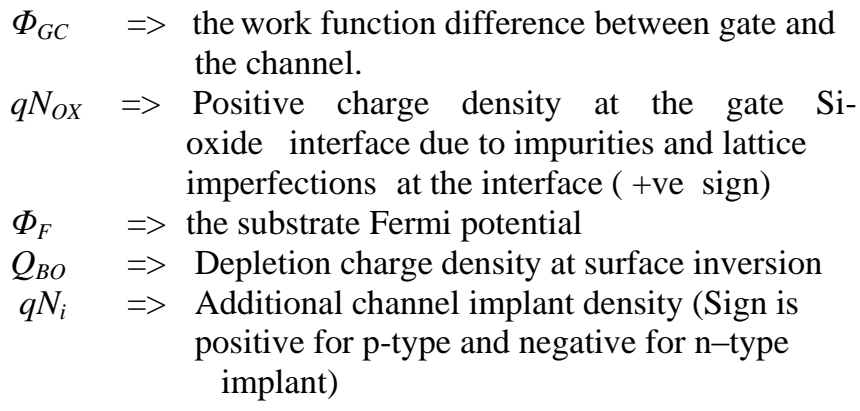

nMOS transistor: $\quad Q_{B O=-\sqrt{2 q N_{A} \epsilon_{S i} \mid-2 \emptyset_{F}}}$

pMOS transistor $\quad Q_{B O}=\sqrt{2 q N_{A} \epsilon_{S i}\left|-2 \emptyset_{F}\right|}$

$$
\begin{gathered}
\emptyset_{G C}=\emptyset_{F}-\emptyset_{F(\text { gate })} \\
\Phi_{\mathrm{F}(\text { gate })}=\left\{\begin{array}{c}
0.55 \mathrm{~V}-\mathrm{N} \text { type polysilicon gate } \\
-0.55 \mathrm{~V}-\mathrm{p} \text { type polysilicon gate } \\
\emptyset_{M}-\text { metal gate }
\end{array}\right.
\end{gathered}
$$

$$
\begin{gathered}
\varepsilon_{O X}=0.34 \mathrm{X} 10^{-12} \mathrm{Fcm}^{-1}, \epsilon_{\mathrm{si}}=1.06 \times 10^{-12} \mathrm{Fcm}^{-1}, \quad C_{O X}=\frac{\varepsilon_{O X}}{t_{O X}} \\
Q_{B=-\sqrt{2 q N_{A} \epsilon_{S i}\left|-2 \emptyset_{F}+V_{S B}\right|}} \\
V_{T}=\emptyset_{G C}-2 \emptyset_{F}-\frac{Q_{B}}{C_{O X}}-\frac{Q_{O X}}{C_{O X}} \\
=\emptyset_{G C}-2 \emptyset_{F}-\frac{Q_{B O}}{C_{O X}}-\frac{Q_{O X}}{C_{O X}}-\frac{Q_{B}-Q_{B O}}{C_{O X}} \\
=V_{T O}-\frac{Q_{B}-Q_{B O}}{C_{O X}}
\end{gathered}
$$

Using (8) and (9) we get, equation for threshold voltage with body bias effect and is given by (10)

$$
V_{t h}=V_{T O}+\gamma\left(\sqrt{\left|-2 \emptyset_{F}+V_{S B}\right|}-\sqrt{\left|2 \emptyset_{F}\right|}\right)
$$

where $\gamma=$ body effect coefficient $=\frac{\sqrt{2 q N_{A} \varepsilon_{s i}}}{C_{O X}}$

Equation (10) is the most familiar threshold equation used for MOSFETs but this has been modeled considering long channel with uniform doping. Thus we see that, the threshold voltage in general depends upon seven factors and they are gate conductor material, gate Oxide material and thickness, substrate doping, Channel Ion Implantation (depending on the dopants type i.e. impurity added p-type (n-type), $V_{\text {th }}$ is made more positive or negative, impurities in Silicon-oxide interface, $\mathrm{Q}_{\mathrm{OX}}$, source bulk voltage or body bias effect, $\mathrm{V}_{\mathrm{SB}}$ and temperature, $\mathrm{T}$. For $\mathrm{V}_{\mathrm{SB}} \neq 0$, threshold voltage is denoted as $\mathrm{V}_{\text {th. }}$.

\section{B. Threshold Voltage for Short Channel Devices}

Equation (11) has been used for derivation and modeling of threshold voltage of short channel devices [4].

$$
\begin{gathered}
V_{t h}=V_{T O}+\gamma\left(\sqrt{\left|2 \emptyset_{F}+V_{S B}\right|}-\sqrt{\left|2 \emptyset_{F}\right|}\right) \\
V_{\mathrm{TO}(\mathrm{SCE})}=V_{\mathrm{TO}}+\Delta V_{\mathrm{TO}}
\end{gathered}
$$

Here, $V_{T O}$ represents long-channel threshold voltage at $\mathrm{V}_{\mathrm{SB}}=0 \mathrm{~V}$ (typical value is $0.7 \mathrm{~V}$ for $\mathrm{NMOS}$ and $-0.7 \mathrm{~V}$ for PMOS) Here, $\Delta \mathrm{V}_{\mathrm{TO}}$ represents a new process variation parameter added to $\mathrm{V}_{\mathrm{TO}}$.

$$
V_{t h(S C E)}=V_{T O(S C E)}+\mathrm{K} 1 \cdot\left(\sqrt{\emptyset_{\mathrm{F}}-V_{\mathrm{SB}}}-\sqrt{\emptyset_{\mathrm{S}}}\right)-\mathrm{K} 2 \cdot \mathrm{V}_{\mathrm{DS}}
$$

where $K 1, K 2$ and $V_{T O}$ are implemented as model parameters for model flexibility. For short channel devices, threshold voltage is also dependent on non-uniform lateral doping, short channel effects causing dependence on channel length and channel width, and drain supply voltage due to DIBL effect. The variation of threshold voltage with respect to SCE and DIBL is modeled as given in (14):

$\Delta V_{t h}(S C E, D I B L)=-\theta_{t h}\left(L_{e f f}\right) \cdot\left[2\left(V_{b i}-\emptyset_{F}\right)+V_{d s}\right]$

where,

$V_{b i}$ is known as the built in voltage of the source/drain junctions and is as given below: 


$$
V_{b i}=\frac{k_{B} T}{q} \operatorname{In}\left(\frac{N_{D E P} N_{S D}}{n_{i}^{2}}\right)
$$

Here, $N_{S D}$ is doping concentration of source/drain diffusion. Thus, it can be concluded that deep-sub-micron levels devices show process and design parameter variations called as parameter variability due to non-uniform doping, variations in channel length and drain voltage.

\section{Variation of $V_{\text {th }}$ with Respect to Length}

Threshold voltage is very important design parameter for any transistor. Since transistors at deep submicron levels show a great variation in $V_{t h}$ due to various factors like DIBL and other short channel effects, it has been the most focused design issue. We see that the SCE are more prominent in short channel devices where silicon di-oxide is used as gate material. Also, threshold voltage is found to be varying with respect to various parameters such as supply voltage $V_{D S}$, channel length $\mathrm{L}$, channel width $\mathrm{W}$ and temperature.Table II shows dependence of threshold voltage on channel length for $180 \mathrm{~nm}$ devices. The threshold voltage along the channel is modified due to the non-uniform substrate charge and is explained by charge channel model in [4].

TABLE II: THRESHOLD DEPENDENCE ON CHANNEL LENGTH IN 180NM

\begin{tabular}{|l|l|l|l|}
\hline \multirow{2}{*}{ SL } & \multicolumn{3}{|c|}{ Channel width $=2 \boldsymbol{\mu}$, temp $=27^{\circ} \mathbf{C}$} \\
\cline { 2 - 4 } & Channel length $(\mathbf{n m})$ & $\boldsymbol{V}_{\text {th }}(\boldsymbol{m} \boldsymbol{V})$ & Leakage power $(\boldsymbol{W})$ \\
\hline 1 & 180 & 0.527 & $2.51 \mathrm{E}-3$ \\
2 & 360 & 0.488 & $1.677 \mathrm{E}-3$ \\
3 & 540 & 0.481 & $1.145 \mathrm{E}-3$ \\
\hline \hline
\end{tabular}

For 180nm technology node we observe that threshold voltage decreases with increasing L leading to more leakage currents in circuits. To design CMOS circuits in order to achieve gain, proper rise time and fall time, bandwidth and high speed operations it is required to size aspect ratio of both NMOS and PMOS. Dependence of $V_{t h}$ on channel length and width can cause dynamic $V_{\text {th }}$ variations causing unpredictable behavior of circuits. The dependency of threshold voltage on $\mathrm{L}$ and $\mathrm{W}$ has been explored in circuit designs in [6]. This dependency of threshold voltage and leakage current on aspect ratio is technology and process dependent.

\section{Drain Induce Barrier Lowering-DIBL}

DIBL effect is the reduction of threshold voltage due to the influence of drain voltage. This effect becomes more prominent as channel length decreases. This effect of DIBL has been well corrected in $45 \mathrm{~nm}$ technology node by replacing the process material polysilicon by high-k dielectric material. Fig. 5 shows transfer characteristics for different drain voltages and it can be observed that threshold voltage reduces as $V_{D S}$ is increased (towards left) between 1-5 volts. Taur \& Ning has come out with this observation in [7], which states that as high drain voltage is applied to a short channel device; the barrier is lowered resulting in further decrease of the threshold voltage.

The maximum threshold voltage for $180 \mathrm{~nm}$ technology is $540 \mathrm{mV}$ (point M5), for $V_{D S}=1 \mathrm{~V}$. The least threshold voltage value in graph is $326 \mathrm{mV}$ for $V_{D S}=5 \mathrm{~V}$ shown by point $\mathrm{M} 1$. These simulations have been carried out room temperature for constant gate voltage, $V_{g s}=1.8 \mathrm{~V}$. The simulations have been carried out using transistor size $\mathrm{W} / \mathrm{L}=2 \mu / 180 \mathrm{~nm}$. The variation of $V_{t h}$, with respect to the drain voltage $V_{D S}$ is caused due to the extension of depletion width below drain region and thus influence of free charge carriers by high drain voltage.

This leads to the formation of close channel between source and drain resulting in flow of more drain current. This impact of drain voltage is called as Drain Induces Barrier Lowering called as DIBL.

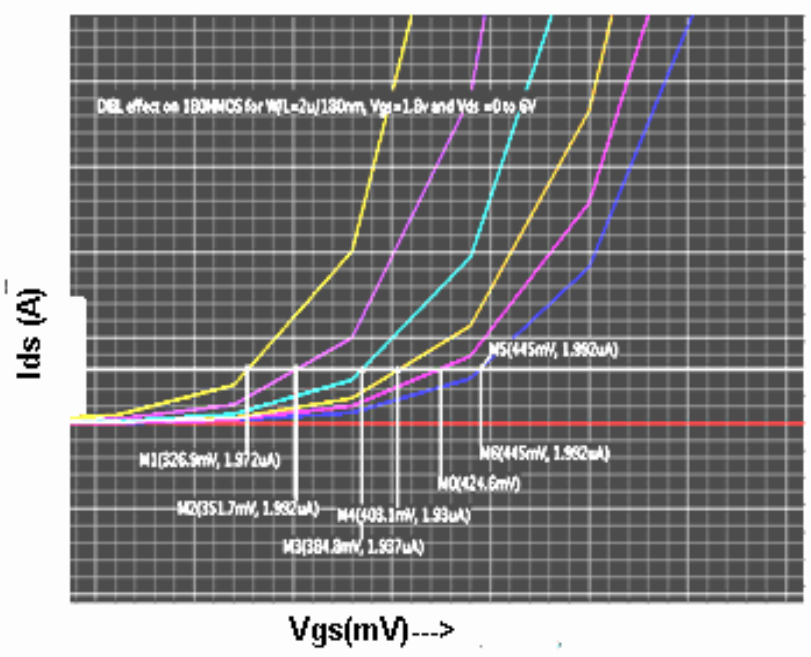

Fig. 5. DIBL effect on threshold voltage (180m).

As seen from Fig. 6, DIBL effect is more in case of $180 \mathrm{~nm}$ node compared to $45 \mathrm{~nm}$. This has been improved by changing the process material between gate and channel. Table III shows the effect of DIBL for both $180 \mathrm{~m}$ and $45 \mathrm{~nm}$ nMOS transistors for range of drain voltage varying from $1 \mathrm{~V}-5 \mathrm{~V}$.

TABLE III: VARIATION OF THREHSOLD VOLTAGE DUE TO DIBL

\begin{tabular}{l|l|l}
\hline \multicolumn{3}{c}{ Temp $=27^{\circ} \mathrm{C}$} \\
\hline \multicolumn{2}{c}{$\boldsymbol{V}_{\boldsymbol{D S}}(\boldsymbol{V})$} & \multicolumn{1}{c}{ Threshold Voltage } \\
$(\mathbf{1 8 0 n m )})$ & $\begin{array}{c}\text { Threshold } \\
\text { Voltage(45nm) }\end{array}$ \\
\hline 0.05 & $608 \mathrm{mV}$ & $590 \mathrm{mV}$ \\
1 & $550 \mathrm{mV}$ & $587 \mathrm{mV}$ \\
2 & $547 \mathrm{mV}$ & $585 \mathrm{mV}$ \\
3 & $442 \mathrm{mV}$ & $584 \mathrm{mV}$ \\
4 & $440 \mathrm{mV}$ & $573 \mathrm{mV}$ \\
5 & $380 \mathrm{mV}$ & - \\
\hline \hline
\end{tabular}

Modeling of short channel devices clearly show that threshold voltage in case of short channel devices depends on two factors apart from $V_{t h}$ as shown in (12) and is given by $V_{T O(\mathrm{SCE})}=V_{T O}+\Delta V_{T O}$. The second term, $\Delta V_{T O}$, represents variability of threshold voltage with respect to short channel effects and Drain Induced barrier Lowering and has been given by (14). These variations in threshold voltage are caused due to change in channel length referred as effective channel length, built in bias voltage between source and drain terminals caused by the presence of lateral and vertical fields and high drain voltage. The doping concentration and process materials also play an important role for this variability. Thus as indicated by ITRS 2011, short channel devices have 
exhausted innovations in process materials and these leakage and power issues may be further improved at design level and architecture level [8].

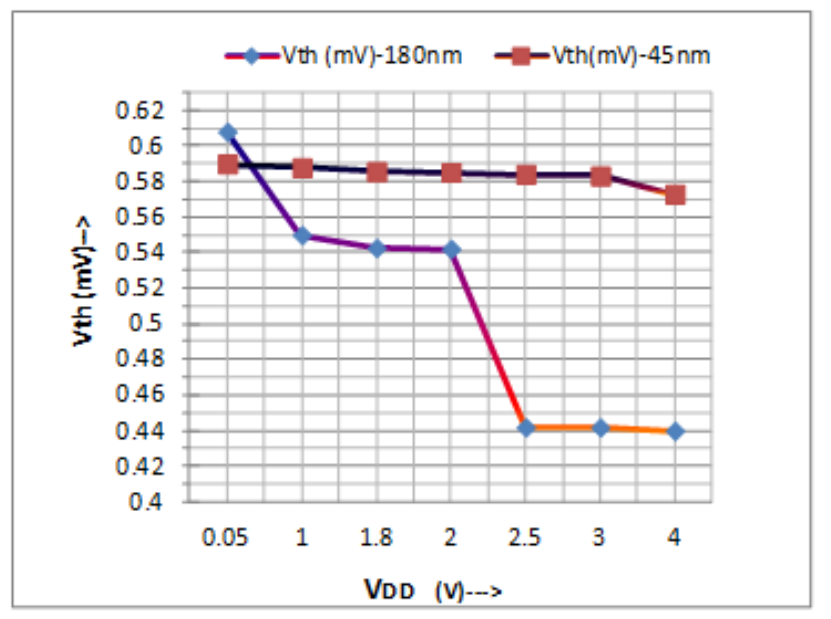

Fig. 6. Threshold variation with supply voltage-DIBL

Fig. 6 indicates improvement in DIBL effect in case of $45 \mathrm{~nm}$ N-Channel MOSFET. This has been achieved by deviation from conventional polysilicon and silicon dioxide gate structures to innovations in process technology by using high-k dielectric material and mestal gate with strain implementation techniques.

\section{CONCLUSION}

This paper presents the results of extensive simulations carried out for $180 \mathrm{~nm}$ and $45 \mathrm{~nm}$ nMOS transistors. This paper presents the results of study of impact of short channel effects and design parameter variations caused in deep submicron devices. The simulation results show that "OFF" state leakage current and subthreshold leakage current are the main source of power consumption in short channel devices.. Though DIBL effect and other short channel effects have been improved in $45 \mathrm{~nm}$ devices by change in process material change of gate, "OFF" state leakage current is much higher compared to upper technology nodes. Also modeling of threshold voltage show that the process design parameter, threshold voltage, was constant in long channel devices. But at deep submicron levels it varies with respect to length, width and supply voltage causing major challenge for CMOS circuit designers. These variations can be mitigated improved at circuit and architecture levels using power gating, multi- $\mathrm{V}_{\text {th }}$ and multi- $\mathrm{V}_{\mathrm{DD}}$ techniques.

\section{ACKNOWLEDGMENT}

Authors would like to extend their thanks to the organizations HTSL, UTL and DSCE for the encouragement and co-operation extended for this research work. First author, Kiran Agarwal would like to thank Dr. A Sreenivasan, director PG studies in Engineering, DSCE, Dr. Siva Y, HOD, UTL technologies and Dr. Sumit Basu for the moral support and technical guidance given to carry out her Ph.D. work.

\section{REFERENCES}

[1] J. Kuhn, K. Kelin, K. Chris, K Anver, and Mark et. al, "Intel's 45nm CMOS Technology," Intel Technology Journal, vol. 12, issue 2, pp. 77-156, June 2008

[2] K. Ahmed and K. Schuegraf, "Transistor wars-rival Architectures face off in a bid to keep Moore's Law alive," IEEE Spectrum, vol. 48, no. 11 , pp. 44-49, November 2011.

[3] J. M. Rabey and M. Pedram, "Low Power Design Methodologies," Kluwer Academic Publishers, Fifth Print 2002, ch. 2, pp. 39.

[4] BSIM 4.6.4 MOSFET Model -Users Manual, T. Hasan M. Wenwei (Morgan) Yang, V. Mohan Dunga, 2009, pp. 39.

[5] M. Pedram, "Minimizing leakage power in CMOS: Technology Issues," presented at USC/EE Centre SI Summer School on Nanoelectronic circuits and tools, July 2008.

[6] K. A. Gupta, V. Venkateswarlu, D. Anvekar, and S. Basu, "The Impact of Channel-Width on Threshold Voltage for Short Channel Devices," in Proc. IEEE Region 10 conference TENCON 2011-Circuits and Systems, Indonesia, November 2011, pp. 715-719.

[7] Y. Taur and T. H. Ning, Fundamentals of Modern VLSI Devices, Cambridge, MA: Cambridge University Press, 1998, pp. 144

[8] Semiconductor Industry Association (SIA), International Roadmap for Semiconductors 2011, Final draft Austin, TX: SEMATECH. (2003). [Online]. Available: http://public.itrs.net

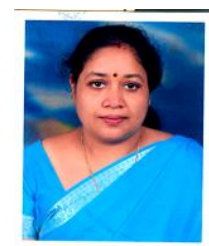

Kiran Agarwal Gupta is Ph.D. scholar at Visvesvaraya Technology University (VTU) and working at Dayananda Sagar College of Engineering (DSCE), Bangalore in the department of Electronics \& Communication as Associate Professor. She obtained Master of technology from VTU in the field of VLSI design and Embedded Systems as specialization. She has published about 20 technical papers and her area of design.

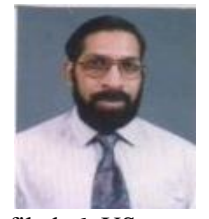

Dinesh K. Anvekar is currently working as Lead, IP and Innovation Initiative at the department of Execution Excellence at Honeywell Technology Solutions Ltd, Bangalore. Dr. Anvekar obtained M.E and Ph.D. degree from Indian Institute of Science and received best Ph.D. thesis award. He has authored one book and 55 technical papers. He received First Plateau Award from IBM and filed 6 US patents. 14 US patents were issued for work done in IBM Solutions. Research during 1998-99, AT\&T Bell Labs during 1993-94 and Lotus works during 2000-04. His area of research interests includes circuits design; Bluetooth related wireless communication and mobile communication.

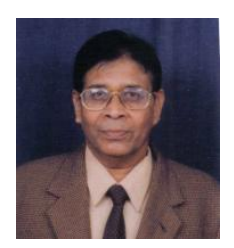

V. Venkateswarlu is currently working as professor and Principal at Vissvesvaraya technology University extension centre at Bangalore. He obtained B.E, M.E and Ph.D. degree from Indian Institute of Science in semiconductor devices. Dr. V. Venkateswarlu has worked for about 25 years with Bharat Electronics Limited. Dr. V. Venkateswarlu has worked at the capacity of research Scientist to Additional General Manager BEL. He was deputed to CDOT for special assignments. IC type BM025 designed and developed by him won ELCINA award for import substitution in the year 1983. He has about two books and 23 technical publications to his credit. His area of research interest includes solid state devices and circuits, Analog circuits and communication solid state devices, low power and circuit design. 